\title{
Comparative analysis of SNP candidates in disparate milk yielding river buffaloes using targeted sequencing
}

Ramesh Menon, Anand B Patel, Chaitanya Joshi

River buffalo (Bubalus bubalis) milk plays an important role in economy and nutritious diet in several developing countries. However, reliable milk-yield genomic markers and their functional insights remain unexposed. Here, we have used a target capture sequencing approach in three economically important buffalo breeds namely: Banni, Jafrabadi and Mehsani, belonging to either high or low milk-yield. Blood samples were collected from the milk-yield/breed balanced group of 12 buffaloes, and whole exome sequencing was performed using Roche 454 GS-FLX Titanium sequencer. Using an innovative approach namely, MultiCom; we have identified high-quality SNPs specific for high and low-milk yield buffaloes. Almost $70 \%$ of the reported genes in QTL regions of milk-yield and milk-fat in cattle were present among the buffalo milk-yield gene candidates. Functional analysis highlighted transcriptional regulation category in the low milk-yield group, and several new pathways in the two groups. Further, the discovered SNP candidates may account for more than half of mammary transcriptome changes in high versus low-milk yielding cattle. Thus, starting from the design of a reliable strategy, we identified reliable genomic markers specific for high and low-milk yield buffalo breeds and addressed possible downstream effects. 
1 Comparative analysis of SNP candidates in disparate milk yielding river buffaloes using targeted

2 sequencing

3 Ramesh Menon ${ }^{1}$, Anand B. Patel ${ }^{1}$ and Chaitanya G. Joshi ${ }^{1 *}$

4 1Department of Animal Biotechnology, College of Veterinary Sciences and Animal Husbandry, Anand 5 Agricultural University, Anand, India.

6

7

8

9

10

$11{ }^{*}$ Corresponding author

12 Prof. Chaitanya G. Joshi

13 Head, Department of Animal Biotechnology

14 College of Veterinary Science \& Animal Husbandry

15 Anand Agricultural University,

16 Anand, Gujarat-388 001 India

17 Phone: +912692 261201

18 email: cgjoshi@aau.in

19 _ cgjoshi@rediffmail.com

20 http://www.omeresearchfacility.in

21 


\section{ABSTRACT}

23 River buffalo (Bubalus bubalis) milk plays an important role in economy and nutritious diet in several developing countries. However, reliable milk-yield genomic markers and their functional insights remain unexposed. Here, we have used a target capture sequencing approach in three economically important buffalo breeds namely: Banni, Jafrabadi and Mehsani, belonging to either high or low milk-yield. Blood samples were collected from the milk-yield/breed balanced group of 12 buffaloes, and whole exome sequencing was performed using Roche 454 GS-FLX Titanium sequencer. Using an innovative approach namely, MultiCom; we have identified high-quality SNPs specific for high and low-milk yield buffaloes. Almost $70 \%$ of the reported genes in QTL regions of milk-yield and milk-fat in cattle were present among the buffalo milk-yield gene candidates. Functional analysis highlighted transcriptional regulation category in the low milk-yield group, and several new pathways in the two groups. Further, the discovered SNP candidates may account for more than half of mammary transcriptome changes in high versus low-milk yielding cattle. Thus, starting from the design of a reliable strategy, we identified reliable genomic markers specific for high and low-milk yield buffalo breeds and addressed possible downstream effects. 


\section{INTRODUCTION}

Dairy farming has been a vital part of agriculture for several thousands of years, and have a high impact in social, health and economic conditions of several Asian countries [www.fao.org]. Notably, India is the largest producer of milk in the world, and buffalo's milk account for about half of India's milk production [www.fao.org]. Milk or milk products form the primary source of fat and protein in an average Indian diet, and buffalo dairy farming is a life sustaining business for millions of small-scale dairy farmers in the country. Economically important Indian buffalo breeds include: Mehsani, Jaffarabadi, Nili-Ravi, Surti, Murrah, Pandharpuri, Banni etc. Despite their multi-faceted significance in countries like India, a few effort has been made so far to improve buffalo breeding programs [1]. It is well understood that milk yield in dairy animals have a major genetic component [2]. In the recent years, genomic selection in cattle using molecular markers such as SNPs has been proved to be highly efficient [3]. A multi-breed analysis will facilitate precise dissection of milk-yield makers and their molecular framework [4-6]. Recently, taking advantage of nextgeneration sequencing with targeted DNA capture technologies are proved to be an efficient and costeffective approach for high-throughput SNP discovery [7].

Here, we designed a whole exome capture custom array that target exons and untranslated regions (UTRs) of the cattle genome to perform whole-exome/targeted sequencing and conducted exome sequencing to identify variants in three recognized milch breeds of river buffalo native to Gujarat state of India viz. Banni, Mehsani, and Jafrabadi breeds. This study design allows us to identify the genetic variants specific for buffalo breeds.

\section{MATERIAL AND METHODS}

\section{Sample collection and genomic DNA extraction}

We recruited 12 water buffaloes, which were biological replicates from three breeds namely Banni, Mehsani and Jafrabadi, belonging to either high or low-milk yield category from the Gujarat state of India (Table 1).

The gDNA was isolated from blood samples using a Qiagen DNeasy Blood and Tissue kit (Qiagen Corp., USA) and the resultant DNA was quantified using Qubit® dsDNA BR Assay (Invitrogen Corp., CA. USA) 
64

65

66

67

and integrity was confirmed by Agarose gels. Blood drawings were conducted in accordance with regulations and prior approval by the institutional animal ethics committee of Anand Agricultural University, Gujarat, India.

67

\section{Probe design, target enrichment and exome sequencing}

We obtained the intended targets (all coding exons, 3'UTR and 5'UTR exons) of cattle (Btau_4.6.1/bos Tau7) from RefGene tables of UCSC genome browser. Further, the custom probe design was performed by NimbleGen (Roche, Germany) which are compatible with Roche GS-FLX Titanium sequencer.

Rapid library for each sample was prepared from $\sim 1 \mu \mathrm{g}$ of gDNA separately and multiplexed according to manufacturer's protocol (Roche, Germany) using high quality DNA. Final libraries were used to set up hybridization reaction at isothermal temperature of $47 \circ \mathrm{C}$ for $68-72$ hours in thermal cycler, with custom designed probes as per manufacturer's protocol (NimbleGen). Captured DNA libraries were quantified spectrophotometrically, and evaluated electrophoretically with high sensitivity DNA assay on Agilent Bioanalyzer 2100 (Agilent). Finally, the libraries were sequenced on Roche 454 GS-FLX Titanium instrument according to manufacturer's protocol (Roche, Germany). The raw sequence data will be deposited in a public repository.

\section{Raw reads filtering, alignment and variant detection}

Raw reads were filtered based on phred quality score $\geq 20$ and length $\geq 50 \mathrm{bp}$ using QTrim tool [8]. The resultant sequence reads were mapped against Bos taurus genome build 4.6.1 using 'bwa-mem' module of BWA v 0.7.5a [9]. Potential PCR duplicates were removed using 'MarkDuplicate' module of Picard Tools. SNPs passing the criteria depth $\geq 5$ and quality score $\geq 30$ were identified using SAMtools, FreeBayes and VarScan tools, through pooled variant calling strategy [10-12]. To deduce the shared (MultiCom) and specific SNPs from each tool, we used VCFtools package v0.1.11 and basic Linux commands [13]. The SNPs belonging to high-yield and low-yield were compared using VCFtools package 
89

90

91

92

93

94

95

96

97

98

99

100

101

102

103

104

105

106

107

108

109

110

111

112

113

114

115

116

117

118

v0.1.11 [13]. The specific SNPs (high or low yield) refers to the SNPs that were present in any of the sample in the given group which are not present or did not pass the SNP calling threshold in the other group.

\section{Annotation and QTL dataset}

The resulting sequence ontology and candidate gene annotation of SNPs specific for high and low yield groups was performed using SnpEff v 3.4 [14]. The candidate gene enrichment analysis was performed in the GeneCodis tool and the enriched pathways and gene ontology terms were identified using Hypergeometric test followed by Benjamini-Hochberg's correction ( $p$-value $<0.01$ ). Cattle QTL candidate genes belonging to milk-yield and milk-fat categories were obtained from the AnimalQTLdb online resource [15].

\section{Mammary gland transcriptome analysis}

From NCBI-GEO database, we obtained the whole gene expression data of 8 samples derived from mammary gland of Holstein Friesen cattle belonging to high or low milk yield (Accession: GSE33680). The gene expression was measured using Agilent-023647 v2 microarray, comprising of 45,220 oligonucleotide probes representing cattle genes. In the dataset, there were four samples in each for high-milk yield and low-milk yield groups. GEO2R implementation of LIMMA ebayes test was used to identify differentially regulated genes between high and low milk yield groups ( $p$-value $<0.01)$.

\section{RESULTS}

\section{Samples and overview of sequence data}

In order to identify SNPs specific for high and low milk-yield in Indian buffalo breeds, we designed two balanced sample groups of healthy Indian buffaloes with prominent difference in milk yields. Animals with fertility issues, inconsistent diets or other known disorders were excluded from the study. The custom designed capture probes based on UCSC RefTable data of Bos taurus genome version 4.6.1/bosTau7 covered 125,679 exons, 14,084 3'UTRs and 16,574 5' UTRs. The sequencing experiment of 12 samples generated about 2.49 gigabases encompassed in $\sim 6.5$ million sequence reads with average length of 380 bases (Table 2). We obtained $\sim 68 \%$ average on-target capture efficiency across samples and 
119

120

121

122

123

124

125

126

127

128

129

130

131

132

133

134

135

136

137

138

139

140

141

142

143

144

145

approximately $98 \%$ of filtered reads were mapped to Bos taurus 4.6 .1 reference sequence (NCBI), with the average per sample depth $\geq 5 X$ (Supplementary table 1 ).

\section{Identification of SNPs specific for high and low milk-yield using MultiCom approach}

We grouped the samples based on milk-yield (high and low milk-yield groups), and performed the SNP analysis separately for each group, using cattle as reference. Initially, we used three variant calling tools namely: SAMtools, FreeBayes and VarScan with the consistent thresholds of base depth $\geq 5$ and quality score $\geq 30$ for all the tools. In the high milk-yield group, SAMtools detected 1.097 million SNPs. FreeBayes and VarScan reported 1.101million and 1.090million SNPs respectively (Figure 1A). Even though high concordances were observed between the outcomes from the three tools, several thousands of SNPs (6.5\% to $9.3 \%$ of output from each tool) were tool-specific or non-reproducible SNP calls (Figure 1A, left Venn diagram). On the other hand, in the low milk-yield group, SAMtools identified 1.389 million SNPs, followed by 1.103 and 1.098 million SNPs respectively by VarScan and FreeBayes. In the low milk-yield group, the tool-specific SNP calls for SAMtools was the same as that of high-production dataset (6.5\%), but a considerable reduction of tool-specific calls was observed for VarScan and FreeBayes (4.6\% and $3.8 \%$ respectively) outputs, even though the total outcome by these two tools were comparable with the high milk-yield group (Figure 1A, left Venn diagram). We hypothesized that the non-reproducible SNPs were potential false positive calls. To test this, we compared the transition/transversion (ts/tv) ratios of SNPs which were tool-specific and shared, separately for the two groups. A highly consistent ts/tv range was observed for the shared SNPs, ranging from 2.61 to 2.65 . On contrary, the tool-specific or non-reproducible SNPs showed a dynamic range of lower ts/tv ratios, ranging from 1.60 to 2.38 (Figure 1B). Strikingly, the difference in the ts/tv ratios were highly significant ( $p$-value <0.005) (Figure 1B). Next, using sequence ontology (SO) annotation by snpEff tool, we compared the regions in the reference genome in which the SNPs are located. Notably, it was observed that the inter-genic region SNPs were almost doubled in case of tool-specific SNP calls, consistently in three tools and the two groups (Figure 1C). Therefore, we considered only those SNPs which were reproduced by at least two tools (hereon called MultiCom approach). There were a total of 1.203 million SNPs in high milk-yield group and 1.315 million SNPs for low 
146 milk-yield group (Figure 1A). Interestingly, in almost all cases, SNP selection through MultiCom approach

147 facilitated an increased discovery rate (up to $20 \%$ ) compared to the outcome from each tool. Finally, we

148 compared MultiCom outcomes in high milk-yield and low milk-yield groups, and have found that 255,741

149 SNPs (21.3\%) were specific for high yield group, and 367,674 SNPs (28.0\%) were specific for low yield

150 group. On the other hand, the 947,646 SNPs were common to the two groups which may be generic SNPs

151 in buffalo, as the reference genome was cattle. 
153 Candidate gene prediction and comparison with milk-yield and milk-fat QTL dataset

154 Using snpEff prediction candidate genes were determined for high and low milk-yield specific SNPs

155 (Supplementary Table 2). The snpEff annotation mapped the 255,741 high yield specific SNPs in 7212

156 genes, and the 367,674 low yield specific SNPs in 8284 coding genes. Even though the SNPs were specific

157 for the two groups, we have found that the 5037 genes were common to high and low milk-yield groups.

158 On the other hand, there were 2175 genes specific for high yield and 3247 for low milk-yield. In order to

159 determine whether these genes were present among the reported milk-yield and milk-fat related genes in

160 QTL region, we obtained the list of candidate genes in milk-yield and milk-fat categories of cattle resources

161 of the animal genome data repository. Remarkably, of 74 milk-yield candidates, 51 genes were present in

162 our results, among which 26 were common to high and low milk-yield group and 17 genes specific for low

163 yield specific genes, and 8 were high specific genes (Figure 2A). On the other hand, of 91 milk-fat

164 candidates, 30 genes were common to high and low-milk yield group, 24 and 15 candidate genes were

165 specific for low and high milk-yield groups respectively (Figure 2B). On the whole, about $70 \%$ of the QTL

166 candidate genes in respective categories belonging to cattle genome annotation were present among the

167 genes identified our study in buffalo breeds.

\section{Enrichment analysis of yield specific candidate genes}

171 Next, we performed the gene ontology (GO) enrichment analysis of the candidate genes, specific for high 172 and low milk-yield groups with the threshold of FDR corrected p-value $<0.01$. In the GO molecular functions category, $8 \mathrm{GO}$ terms were enriched in high milk-yield group. On the other hand, 21 terms were enriched

174 in low-milk yield group, among which 4 terms were common to GO terms belonging to high milk-yield group

175 [Figure 3]. The common terms were metal ion binding, nucleotide binding, zinc ion binding and cytokine activity. Interestingly, several GO terms in the low milk-yield category which were related to transcriptional regulation, such as sequence-specific DNA binding transcription factor activity (GO:0003700), sequencespecific DNA binding (GO:0043565) and transcription regulatory region DNA binding (GO:0044212) [Figure terms, which were specific for low-milk yield group [Figure 3C]. 
181 In order to obtain the clues about the functional consequences of the candidate genes specific for high and 182 low-yield specific groups, we performed the KEGG pathway enrichment analysis using Genecodis tool.

183 There were 15 pathways enriched for the high yield specific group and 32 pathways for the low-yield specific

184 group [Supplementary table 3]. However, we have noticed that several of the pathways were related to 185 human diseases. Further, after excluding human disease specific pathways, there were 8 pathways present 186 in high-yield group and 19 pathways for the low milk yield group [Table 3]. The most significant pathways 187 in the low milk-yield group were Oxidative phosphorylation, Toll-like receptor signalling, cytokine-cytokine receptor interaction etc. On the other hand, the most significant among the high-yield group were Jak-STAT signalling pathway, Wnt signalling pathway, ErbB signalling pathway etc. Surprisingly, Toll-like receptor signalling and MAPK signalling pathway were enriched in both high and low milk-yield groups by distinct set of genes falling in the same pathway [Supplementary figures 1-3].

\section{Comparison of buffalo milk-yield specific candidate genes with milk-yield DEG (Differentially} Expressed Genes) in cattle

Next, we hypothesized that the SNPs in the candidate gene may exert changes in the gene expression that could have an effect on milk yield. In order to verify this, we obtained the mammary cell transcriptome dataset belonging to high and low milk-yielding cattle (see materials and methods). LIMMA analysis between the high milk-yield and low milk-yield group detected 1056 annotated differentially expressed genes, in which 579 gene were up-regulated and 477 genes were down-regulated among the low milk-yield samples (Figure 4, Supplementary table 4). Quite interestingly, it was found that 582 out of 1056 DEG were buffalo SNP candidate genes in low yield specific, high yield specific or common to both groups (Figure 4). Among these 582 overlapping DEG, 196 genes (118 up-regulated and 78 down-regulated) belonged to low yield specific group, 139 genes (68 up-regulated and 71 down regulated) were high yield specific group. On the other hand, 247 DEG (147 up-regulated and 100 down-regulated in low milk yield) were common to high and low milk-yield groups. However, we didn't observe any trend in the direction of fold change with respect to milk-yield group. Next, in order to assess whether the overlap between the DEG and the SNP candidates are not due to random chance, we performed Hypergeometric tests on each category viz. low yield specific, high yield specific and common. Interestingly, in all categories the p-values were below 
209 0.0001. Thus, considering that SNP candidate genes accounted more than half of the DEG in a cross-

210 species comparison, we propose that buffalo SNP candidates potentially influence downstream gene

211 expression changes to a large extend.

\section{DISCUSSION}

213 In India, milk or milk products form one of the primary source of nutrition in an average diet, and water 214 buffalo's milk contribute to about 55\% country's total milk production [16]. In this study, using Roche 454

215 GS-FLX Titanium sequencer we performed exome sequencing of replicated samples of three Indian buffalo

216 breeds belonging to high or low milk-yield groups. The contrast between high and low-milk yield across

217 breeds were about 3.7 fold, which was not influenced by disease, fertility or diet factors. The sequencing

218 experiment generated about $2.49 \mathrm{~Gb}$ of data, and the reads were mapped to cattle genome, as the complete

219

220

221

222 assembly of water buffalo whole genome sequence is not available yet. Initially, this resulted in high discovery rate of SNPs compared to the previously reports $[17,18]$. However, the number of SNPs have markedly reduced when we filtered out those which are specific for low and high milk-yielding group. It has been recently reported that there are indigenous bias for each SNP calling algorithm which causes disagreement of results across software tools $[19,20]$. However, only few efforts has be taken to assess this aspect systematically [20]. In this study, we have used three algorithms for SNP calling and have selected those SNP which were detected by at least two tools. Initially, in order to assess potential true positive calls, we have applied the widely accepted ts/tv metrics [21]. According to the 1000 genomes project, the expected ts/tv ratio in whole-genome sequencing is about 2.10 , and for exome target regions ranges from 2.6 to 3.5 (http://www.1000genomes.org/). We have not only observed a lower ts/tv ratio in the non-reproducible SNP calls, but also a varying range of ts/tv values. In addition, the SNP calls from intergenic region were almost doubled in case of non-reproducible SNPs. Overall, we propose the MultiCom approach as a simple but effective strategy to identify reliable SNPs, without decreasing overall discovery rate.

After determining the SNPs specific for high and low milk-yield groups, it is important to understand its possible consequences by extending the analysis at gene level, which is a very useful strategy to obtain the possible molecular framework hosted by the SNPs [22-24]. Towards this, we focused on the genes in 
236

237

238

239

240

241

242

which SNPs fall in their exonic region. Initially, we have seen that majority of the milk-yield and milk-fat QTL candidate genes are found among the discovered group. The SNP candidate genes specifically found in the low-yield specific group includes ABCG2, CSN2, CSN3, IL12RB2, SRC, STAT5B etc. The classic milk proteins such as CSN2, CSN3 (members of casein family), STAT5B etc. are prominent indicators of milk yield parameters [25]. Interestingly, in the low-yield specific group, there were several genes related to immunological properties of milk. For example, $A B C G 2$ gene is responsible for the active secretion of clinically and toxicologically relevant substrates into the milk and soluble CD14 in colostrum and milk acts as a sentinel molecule and an immune modulator, which provide innate responses against bacterial infections in the calf [26] [27]. IL12RB2 gene is another example in this category. Thus the low-yield specific group not only contained genes directly implicated in milk yield, but also comprised of genes that contribute to major immunological properties of milk. On the other hand, high-yield specific candidate genes included ARFGEF1, GH1, DGAT1, IRF9, PRL etc., which are hallmark genes for milk yield in cattle. Interestingly, Shi and colleges have recently shown that the polymorphism of several of these genes affects the milk composition in water different buffalo breeds [28].

Gene Ontology enrichment analysis showed several transcriptional regulation related terms in the low-milk yield group, and we highlighted the 140 genes responsible for this observation. Recently, a gene network analysis of human mammary transcriptome data highlighted crucial nodes such as $\mathrm{NR} 1 \mathrm{H} 3$ and PPARA genes [29]. Notably, these genes are present in our results, and it was reported that PPARA and its target genes has been shown to be involved in fatty acid uptake/oxidation, and promotes energy balance during early lactation in cattle [30].

The pathway enrichment analysis pointed towards several known pathways implicated in mammary biology such as JAK-STAT signalling, Wnt signalling, epidermal growth factor related pathways (ErbB signalling, VEGF signalling) etc. [31-34]. In the low milk-yield specific group, Oxidative phosphorylation pathway was the most enriched pathway, which may contribute to the energy balance during different functional states of mammary gland [35]. Interestingly, there were two pathways common to high and low milk-yield groups. Considering that the input genes for the pathway analysis were different, Toll-like receptor signalling and MAP kinase signalling pathways were enriched by diverse set of genes belonging to the two 
263

264

265

266

267

268

269

270

271

272

273

274

275

276

277

278

279

280

281

282

283 groups. Very interestingly, O'Neill and colleagues have extensively reviewed Toll-like receptor signalling pathway, and suggested the possible dual outcomes of this pathway [36]. Two important families of transcription factors that are activated downstream of TLR signalling are nuclear factor-KB (NF-KB) and the interferon-regulatory factors (IRF3/7), and the major consequence of NFKB mediated signalling is the production of interleukins, while the IRFs induces type I interferon [36]. Notably, RELA (a member of NFKB complex) was present among low-specific candidates and IRF3 was present in the high milk-yield specific group. Of note, TLR-4 mediated excess interleukin production has shown to cause lactation insufficiency in mice [37]. On the other hand, increased interferon level was observed in breastfeeding infants compared to non-breast feeding group, which confers enhanced protection against viral infection for the infant [38]. However, more studies need to be performed in this direction, as evidenced by limited number of studies.

Comparison of gene expression and SNP candidate genes offers a great extent of biological insights[39]. Recently, Cui and colleagues observed differential gene expression in various susceptibility genes related to milk protein and fat level in Holstein friesen cattle [40]. In our case, the buffalo SNP data were identified based on cattle as the reference genome, and the gene expression data were from cattle. However, we excluded the putative generic SNPs in buffalo breeds (found common to both high and lowyield groups) at the initial stage of analysis which makes the comparison relevant, even though the genome and transcriptome data are from buffalo and cattle species respectively. In the comparison, we found a strong presence of high or low-yield specific SNP containing genes among the DEG. This observation not only underlines the strategy of comparison but also provides positive indication for the reliability of the identified SNPs which need to be investigated in additional datasets. 
285

286

287

288

289

290

291

292

293

294

295

296

297

298

299

300

301

302

\section{CONCLUSION}

In this study, we have highlighted issues related to SNPs detection using a single tool and propose selection of reproducible SNPs using Multicom approach, which facilitates detection of reproducible SNPs with a higher discovery rate. Focussed analysis on the high and low milk-yield SNPs in buffalo breeds, uncovered SNPs specific for high or low milk yield. A substantial number of reported genes in QTL region of cattle genome is present in results. Further, the enrichment analysis uncovered several transcriptional regulator candidates in low-milk yield group. Surprisingly, pathway analysis shared pathways like TLR receptor signalling, along with several known and specific pathways in high and low-milk yield groups. Finally, SNP candidate genes in buffalo breeds account for were splendidly found in differentially expressed genes from the cattle species. On the whole, these results not only highlighted SNPs related to high and low-yield buffalo, but also sheds light to methodological improvement, functional insights and cross species genome-phenome comparison. Of course, outcomes of this study need to be validated in independent samples which in turn contribute for the better genomic selection in buffalo breeds. 


\section{REFERENCES}

305

306

307

308

309

310

311

312

313

314

315

316

317

318

319

320

321

322

323

324

325

326

327

328

329

330

331

332

333

334

335

336

337

338

339

340

341

342

343

344

345

346

347

348

1. Singh B, Chauhan MS, Singla SK, Gautam SK, Verma V, Manik RS, Singh AK, Sodhi M, Mukesh M (2009) Reproductive biotechniques in buffaloes (Bubalus bubalis): status, prospects and challenges. Reprod Fertil Dev 21: 499-510.

2. Cole JB, VanRaden PM, O'Connell JR, Van Tassell CP, Sonstegard TS, Schnabel RD, Taylor JF, Wiggans GR (2009) Distribution and location of genetic effects for dairy traits. J Dairy Sci 92: 2931-2946.

3. Berglund B (2008) Genetic improvement of dairy cow reproductive performance. Reprod Domest Anim 43 Suppl 2: 89-95.

4. Goddard ME, Hayes BJ (2009) Mapping genes for complex traits in domestic animals and their use in breeding programmes. Nat Rev Genet 10: 381-391.

5. Melka MG, Schenkel FS (2012) Analysis of genetic diversity in Brown Swiss, Jersey and Holstein populations using genome-wide single nucleotide polymorphism markers. BMC Res Notes 5: 161.

6. Olson KM, VanRaden PM, Tooker ME (2012) Multibreed genomic evaluations using purebred Holsteins, Jerseys, and Brown Swiss. J Dairy Sci 95: 5378-5383.

7. Hirano $T$, Kobayashi $N$, Matsuhashi $T$, Watanabe $D$, Watanabe $T$, Takasuga $A$, Sugimoto $M$, Sugimoto $Y$ (2013) Mapping and exome sequencing identifies a mutation in the IARS gene as the cause of hereditary perinatal weak calf syndrome. PLoS One 8: e64036.

8. Schmieder R, Edwards R (2011) Quality control and preprocessing of metagenomic datasets. Bioinformatics 27: 863-864.

9. Li H, Durbin R (2010) Fast and accurate long-read alignment with Burrows-Wheeler transform. Bioinformatics 26: 589-595.

10. Li H, Handsaker B, Wysoker A, Fennell T, Ruan J, Homer N, Marth G, Abecasis G, Durbin R; 1000 Genome Project Data Processing Subgroup (2009) The Sequence Alignment/Map format and SAMtools. Bioinformatics 25: 2078-2079.

11. Garrison E, Marth G (2012) Haplotype-based variant detection from short-read sequencing. arXiv 1207.3907.

12. Koboldt DC, Zhang Q, Larson DE, Shen D, McLellan MD, Lin L, Miller CA, Mardis ER, Ding L, Wilson RK (2012) VarScan 2: somatic mutation and copy number alteration discovery in cancer by exome sequencing. Genome Res 22: 568-576.

13. Danecek P, Auton A, Abecasis G, Albers CA, Banks E, DePristo MA, Handsaker RE, Lunter G, Marth GT, Sherry ST, McVean G, Durbin R; 1000 Genomes Project Analysis Group (2011) The variant call format and VCFtools. Bioinformatics 27: 2156-2158.

14. Cingolani P, Platts A, Wang le L, Coon M, Nguyen T, Wang L, Land SJ, Lu X, Ruden DM (2012) A program for annotating and predicting the effects of single nucleotide polymorphisms, SnpEff: SNPs in the genome of Drosophila melanogaster strain w1118; iso-2; iso-3. Fly (Austin) 6: 80-92.

15. Hu Z-L, Park CA, Wu X-L, Reecy JM (2013) Animal QTLdb: an improved database tool for livestock animal QTL/association data dissemination in the post-genome era. Nucleic acids research 41: D871-D879.

16. Michelizzi VN, Dodson MV, Pan Z, Amaral ME, Michal JJ, McLean DJ, Womack JE, Jiang Z (2010) Water buffalo genome science comes of age. Int J Biol Sci 6: 333-349.

17. Jansen S, Aigner B, Pausch H, Wysocki M, Eck S, Benet-Pagès A, Graf E, Wieland T, Strom TM, Meitinger T, Fries R. (2013) Assessment of the genomic variation in a cattle population by resequencing of key animals at low to medium coverage. BMC Genomics 14: 446.

18. Georges M (2014) Towards sequence-based genomic selection of cattle. Nat Genet 46: 807-809. 
19. Pabinger S, Dander A, Fischer M, Snajder R, Sperk M, Efremova M, Krabichler B, Speicher MR, Zschocke J, Trajanoski Z (2014) A survey of tools for variant analysis of next-generation genome sequencing data. Brief Bioinform 15: 256-278.

20. Yi M, Zhao Y, Jia L, He M, Kebebew E, Stephens RM (2014) Performance comparison of SNP detection tools with illumina exome sequencing data--an assessment using both family pedigree information and sample-matched SNP array data. Nucleic Acids Res 42: e101.

21. DePristo MA, Banks E, Poplin R, Garimella KV, Maguire JR, Hartl C, Philippakis AA, del Angel G, Rivas MA, Hanna M, McKenna A, Fennell TJ, Kernytsky AM, Sivachenko AY, Cibulskis K, Gabriel SB, Altshuler D, Daly MJ (2011) A framework for variation discovery and genotyping using nextgeneration DNA sequencing data. Nat Genet 43: 491-498.

22. Lehne B, Lewis CM, Schlitt T (2011) From SNPs to genes: disease association at the gene level. PLoS One 6: e20133.

23. Menon R, Farina C (2011) Shared molecular and functional frameworks among five complex human disorders: a comparative study on interactomes linked to susceptibility genes. PLoS One 6: e18660.

24. Shastry BS (2009) SNPs: impact on gene function and phenotype. Methods Mol Biol 578: 3-22.

25. Lee HJ, Kim J, Lee T, Son JK, Yoon HB, Baek KS, Jeong JY, Cho YM, Lee KT, Yang BC, Lim HJ, Cho K, Kim TH, Kwon EG, Nam J, Kwak W, Cho S, Kim H (2014) Deciphering the genetic blueprint behind Holstein milk proteins and production. Genome Biol Evol 6: 1366-1374.

26. Olsen HG, Nilsen H, Hayes B, Berg PR, Svendsen M, Lien S, Meuwissen T (2007) Genetic support for a quantitative trait nucleotide in the ABCG2 gene affecting milk composition of dairy cattle. BMC Genet 8: 32.

27. Ibeagha-Awemu EM, Lee JW, Ibeagha AE, Zhao X (2008) Bovine CD14 gene characterization and relationship between polymorphisms and surface expression on monocytes and polymorphonuclear neutrophils. BMC Genet 9: 50.

28. Shi DS, Wang J, Yang Y, Lu FH, Li XP, Liu QY (2012) DGAT1, GH, GHR, PRL and PRLR polymorphism in water buffalo (Bubalus bubalis). Reprod Domest Anim 47: 328-334.

29. Mohammad MA, Haymond MW (2013) Regulation of lipid synthesis genes and milk fat production in human mammary epithelial cells during secretory activation. Am J Physiol Endocrinol Metab 305: E700-716.

30. Schlegel G, Keller J, Hirche F, Geissler S, Schwarz FJ, Ringseis R, Stangl GI, Eder K (2012) Expression of genes involved in hepatic carnitine synthesis and uptake in dairy cows in the transition period and at different stages of lactation. BMC Vet Res 8: 28.

31. Watson CJ, Burdon TG (1996) Prolactin signal transduction mechanisms in the mammary gland: the role of the Jak/Stat pathway. Rev Reprod 1: 1-5.

32. Rossiter H, Barresi C, Ghannadan M, Gruber F, Mildner M, Födinger D, Tschachler E (2007) Inactivation of VEGF in mammary gland epithelium severely compromises mammary gland development and function. FASEB J 21: 3994-4004.

33. Hardy KM, Booth BW, Hendrix MJ, Salomon DS, Strizzi L (2010) ErbB/EGF signaling and EMT in mammary development and breast cancer. J Mammary Gland Biol Neoplasia 15: 191-199.

34. Turashvili G, Bouchal J, Burkadze G, Kolar Z (2006) Wnt signaling pathway in mammary gland development and carcinogenesis. Pathobiology 73: 213-223.

35. Nelson WL, Butow RA, Ciaccio El (1962) Oxidative phosphorylation in guinea pig mammary gland mitochondria during various functional states. Arch Biochem Biophys 96: 500-505.

36. O'Neill LA, Golenbock D, Bowie AG (2013) The history of Toll-like receptors - redefining innate immunity. Nat Rev Immunol 13: 453-460. 
395

396

397

398

399

400

401

402

403

404

405

406

407

37. Glynn DJ, Hutchinson MR, Ingman WV (2014) Toll-like receptor 4 regulates lipopolysaccharideinduced inflammation and lactation insufficiency in a mouse model of mastitis. Biol Reprod 90: 91.

38. Melendi GA, Coviello S, Bhat N, Zea-Hernandez J, Ferolla FM, Polack FP (2010) Breastfeeding is associated with the production of type I interferon in infants infected with influenza virus. Acta Paediatr 99: 1517-1521.

39. Quan B, Qi X, Yu Z, Jiang Y, Liao M, Wang G, Feng R, Zhang L, Chen Z, Jiang Q, Liu G (2014) Pathway analysis of genome-wide association study and transcriptome data highlights new biological pathways in colorectal cancer. Mol Genet Genomics.

40. Cui X, Hou Y, Yang S, Xie Y, Zhang S, Zhang Y, Zhang Q, Lu X, Liu GE, Sun D (2014) Transcriptional profiling of mammary gland in Holstein cows with extremely different milk protein and fat percentage using RNA sequencing. BMC Genomics 15: 226. 
Figure 1: SNPs discovery using Multicom approach: (A) In the upper panel, left and the right Venn diagrams shows the MultiCom SNP discovery in the high and low production respectively. tools are given. In the upper panel, the numbers given in uncoloured region inside the Venn diagram is the number of non-reproducible or tool-specific SNPs. Multicom approach identified 1.203 million and 1.315 million SNPs respectively in high and low production group. The lower panel Venn diagram shows the number of shared and specific SNPs in high and low yield groups. (B) Comparison of ts/tv ratios in tool-specific and shared SNPs outputted by each of the three tools in high and low yield groups. The difference in ts/tv ratio is statistically significant (p.val <0.005) (C) Percentage of intergenic SNPs detected by each of the three tools in high and low yield groups.

Figure 2: Gene level comparison of SNPs specific in high and low milk-yield groups with QTL dataset: (A) Overlapping genes in milk-yield category. The first column represents high and low milk yield specific SNPs located in 26 common genes. The second and third column respectively indicates the 17 low-milk yield specific and 8 high-milk yield specific candidate genes (B) Overlapping genes in milk-fat category. The first column represents high and low milk yield specific SNPs located in 30 common candidate genes. The second and third column respectively indicates the 24 low-milk yield specific and 15 high-milk yield specific candidate genes.

Figure 3: Gene ontology enrichment analysis of high and low specific gene candidates: (A) Twenty one gene ontology (molecular functions) terms enriched in low-yield specific group. The number of hits associated with each term along with the FDR corrected p-value is given. (B) Eight gene ontology (molecular functions) terms enriched in high-yield specific group. (C) The transcriptional regulators (140 genes) highlighted by the $\mathrm{GO}$ analysis in low-yield group. 
from buffalo breeds: The upper panel shows the workflow for the derivation of the DEG in high and low milk-yielding cattle using Agilent microarrays. The Venn diagram highlights the 582 DEG (55.1\% of total) which are discovered SNP candidate genes discovered by our multi-breed study in buffaloes. The colored bar in indicates the group of SNP candidates in each category. Green color indicates 247 candidate genes common to high and low-yield specific groups, derived from specific SNPs in both the groups. Yellow and red color indicates the specific high-yield (139) and low-yield (196) candidate genes respectively. The overlap of the DEG and in each of these category were found to statistically significant in Hypergeometric test (p.val < 0.005) 


\section{Table $\mathbf{1}$ (on next page)}

Table 1 
1 Table 1: Summary of samples recruited for the analysis.

\begin{tabular}{|c|c|c|c|}
\hline Group & Sample ID & Breed & Total milk yield (liters) \\
\hline \multirow{6}{*}{ Low milk yield } & BLP1 & \multirow{2}{*}{ Banni } & 1680 \\
\hline & BLP2 & & 1400 \\
\hline & MLP2 & \multirow{2}{*}{ Mehsani } & 1401 \\
\hline & MLP5 & & 1449 \\
\hline & JLP1 & \multirow{2}{*}{ Jafarabadi } & 571 \\
\hline & JLP2 & & 583 \\
\hline \multirow{6}{*}{ High milk yield } & BHP1 & \multirow{2}{*}{ Banni } & 6440 \\
\hline & BHP2 & & 5880 \\
\hline & MHP1 & \multirow{2}{*}{ Mehsani } & 4104 \\
\hline & MHP2 & & 4091 \\
\hline & JHP1 & \multirow{2}{*}{ Jafarabadi } & 3186 \\
\hline & JHP2 & & 2947 \\
\hline
\end{tabular}

2 


\section{Table 2 (on next page)}

Table 2 
1 Table 2: Summary of sequence data.

\begin{tabular}{|c|c|c|c|c|c|c|c|c|}
\hline \multirow[b]{2}{*}{$\begin{array}{l}\text { Sample } \\
\text { ID }\end{array}$} & \multicolumn{3}{|c|}{ Raw dataset } & \multicolumn{5}{|c|}{ Filtered dataset } \\
\hline & $\begin{array}{l}\text { Total } \\
\text { Reads }\end{array}$ & $\begin{array}{l}\text { Base } \\
\text { count } \\
(\mathrm{Mb})\end{array}$ & $\begin{array}{l}\text { Mean } \\
\text { Read } \\
\text { Length }\end{array}$ & $\begin{array}{l}\text { No. of } \\
\text { Reads }\end{array}$ & $\begin{array}{l}\text { Base } \\
\text { count } \\
(\mathrm{Mb})\end{array}$ & $\begin{array}{l}\text { Mean } \\
\text { Read } \\
\text { Length }\end{array}$ & $\%$ passed & $\begin{array}{c}\text { Target } \\
\text { covered } \\
(\%)\end{array}$ \\
\hline BLP1 & 468,655 & 179.2 & 382 & 434,193 & 149.6 & 344 & 92.6 & 70.6 \\
\hline BLP2 & 544,069 & 206.1 & 379 & 503,649 & 173 & 343 & 92.6 & 71.7 \\
\hline BHP1 & 604,742 & 229.7 & 380 & 555,099 & 195 & 351 & 91.8 & 63.3 \\
\hline BHP2 & 629,606 & 240.7 & 382 & 580,983 & 204.8 & 352 & 92.3 & 65.5 \\
\hline MLP2 & 573,466 & 213 & 371 & 530,089 & 176.3 & 332 & 92.4 & 67.2 \\
\hline MLP5 & 437,957 & 165.4 & 378 & 407,996 & 137.1 & 336 & 93.2 & 73.3 \\
\hline MHP1 & 544,403 & 197.3 & 362 & 498,613 & 163.2 & 327 & 91.6 & 70.2 \\
\hline MHP2 & 657,400 & 238.4 & 363 & 602,554 & 197.3 & 327 & 91.7 & 62.8 \\
\hline JLP1 & 632,961 & 249 & 393 & 594,251 & 213.9 & 359 & 93.9 & 65.3 \\
\hline JLP2 & 576,284 & 227.3 & 394 & 541,456 & 195.4 & 360 & 94 & 66.3 \\
\hline JHP1 & 447,055 & 172.3 & 385 & 413,569 & 145.1 & 350 & 92.5 & 76.4 \\
\hline JHP2 & 445,066 & 180 & 404 & 413,702 & 151.7 & 366 & 93 & 74.3 \\
\hline & $\begin{array}{c}6,561,664 \\
\text { (total) }\end{array}$ & $\begin{array}{l}2,498 \\
\text { (total) }\end{array}$ & $\begin{array}{c}381 \\
\text { (average) }\end{array}$ & $\begin{array}{c}6,076,154 \\
\text { (total) }\end{array}$ & $\begin{array}{l}2,102 \\
\text { (total) }\end{array}$ & $\begin{array}{c}346 \\
\text { (average) }\end{array}$ & $\begin{array}{c}92.6 \\
\text { (average) }\end{array}$ & $\begin{array}{c}67.8 \\
\text { (average) }\end{array}$ \\
\hline
\end{tabular}

2 


\section{Table $\mathbf{3}$ (on next page)}

Table 3 
1 Table 3: Pathways enriched in low and high milk-yield groups

\begin{tabular}{|c|c|c|c|}
\hline KEGG Pathways & Group & Genes & FDR p-val \\
\hline Oxidative phosphorylation & $\mathrm{L}$ & 33 & $1.53 \mathrm{E}-07$ \\
\hline Toll-like receptor signalling pathway * & $\begin{array}{l}\mathrm{L} \\
\mathrm{H}\end{array}$ & $\begin{array}{l}20 \\
13\end{array}$ & $\begin{array}{l}3.39 \mathrm{E}-05 \\
4.54 \mathrm{E}-03\end{array}$ \\
\hline Cytokine-cytokine receptor interaction & $\mathrm{L}$ & 26 & 1.08E-04 \\
\hline Jak-STAT signalling pathway & $\mathrm{H}$ & 18 & 1.96E-04 \\
\hline Melanogenesis & $\mathrm{L}$ & 14 & $6.75 \mathrm{E}-04$ \\
\hline Spliceosome & L & 21 & 9.81E-04 \\
\hline Viral myocarditis & L & 11 & $1.03 E-03$ \\
\hline Wnt signaling pathway & $\mathrm{H}$ & 17 & $1.91 \mathrm{E}-03$ \\
\hline RIG-I-like receptor signaling pathway & L & 12 & 2.99E-03 \\
\hline Osteoclast differentiation & L & 17 & $3.25 \mathrm{E}-03$ \\
\hline Toxoplasmosis & L & 17 & 3.25E-03 \\
\hline Adipocytokine signaling pathway & L & 12 & $3.40 \mathrm{E}-03$ \\
\hline Protein digestion and absorption & L & 10 & 3.91E-03 \\
\hline ErbB signaling pathway & $\mathrm{H}$ & 10 & 4.14E-03 \\
\hline Natural killer cell mediated cytotoxicity & $\mathrm{H}$ & 13 & 4.36E-03 \\
\hline Leukocyte transendothelial migration & $\mathrm{H}$ & 14 & 4.43E-03 \\
\hline Cell cycle & L & 17 & 4.87E-03 \\
\hline MAPK signaling pathway * & $\begin{array}{l}\mathrm{L} \\
\mathrm{H}\end{array}$ & $\begin{array}{l}27 \\
21\end{array}$ & $\begin{array}{l}5.01 \mathrm{E}-03 \\
5.28 \mathrm{E}-03\end{array}$ \\
\hline Vasopressin-regulated water reabsorption & L & 9 & $6.02 \mathrm{E}-03$ \\
\hline VEGF signaling pathway & $\mathrm{H}$ & 10 & 6.79E-03 \\
\hline Collecting duct acid secretion & L & 7 & 7.38E-03 \\
\hline Axon guidance & L & 15 & 7.50E-03 \\
\hline Apoptosis & L & 13 & 7.56E-03 \\
\hline Cell adhesion molecules (CAMs) & L & 15 & 8.50E-03 \\
\hline Protein processing in endoplasmic reticulum & L & 20 & $9.14 \mathrm{E}-03$ \\
\hline
\end{tabular}

2 L= Low milk-yield, $\mathrm{H}=$ High milk-yield. *Common pathways in $\mathrm{L}$ and $\mathrm{H}$ groups. 


\section{1}

Figure 1

SNPs discovery using Multicom approach: $(A)$ In the upper panel, left and the right Venn diagrams shows the MultiCom SNP discovery in the high and low production respectively. The total number of high quality SNPs detected by SAMtools, VarScan and Freebayes tools are given. In the upper panel, the numbers given in uncoloured region inside the Venn diagram is the number of non-reproducible or tool-specific SNPs. Multicom approach identified 1.203 million and 1.315 million SNPs respectively in high and low production group. The lower panel Venn diagram shows the number of shared and specific SNPs in high and low production groups. (B) Comparison of ts/tv ratios in tool-specific and shared SNPs outputted by each of the three tools in high and low production groups. The difference in ts/tv ratio is statistically significant (p.val <0.005) (C) Percentage of intergenic SNPs detected by each of the three tools in high and low production groups.

A

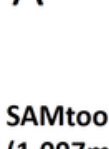

(1.097m)
High yield

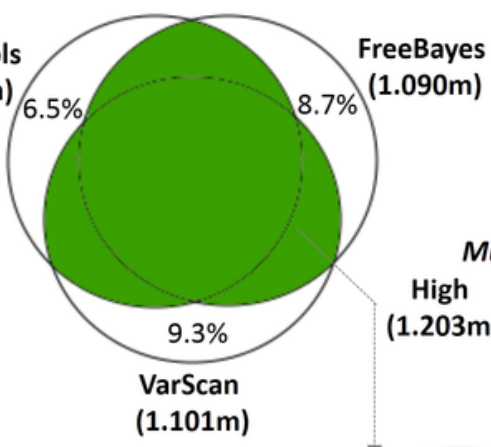

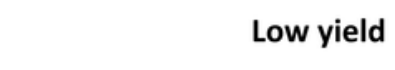

SAMtools

(1.389m)

$1.389 \mathrm{~m})$

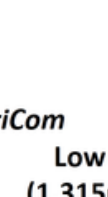

$(1.315 \mathrm{~m})$

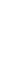

High production specific SNPs

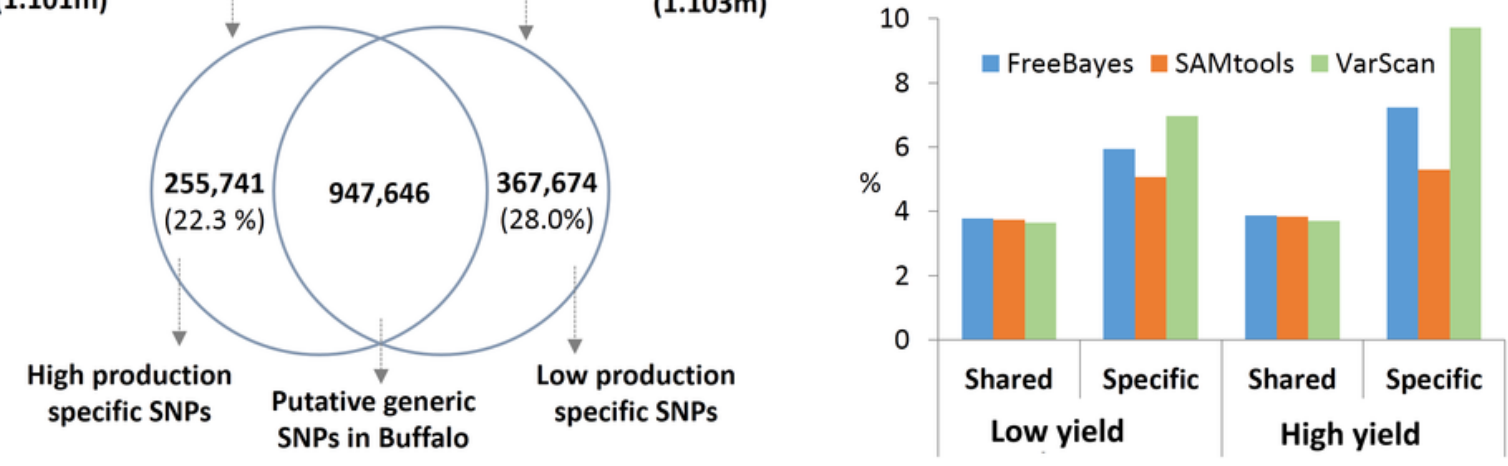

Low yield

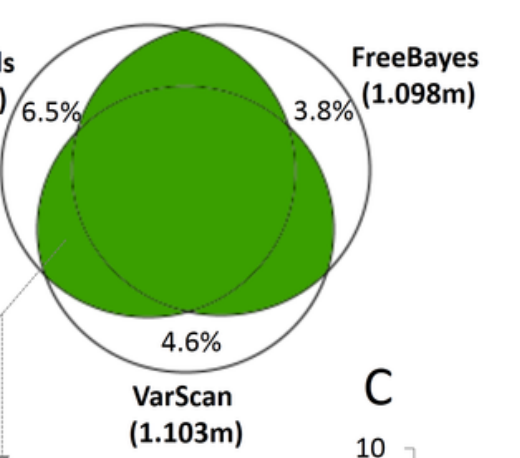

$(1.103 \mathrm{~m})$
B

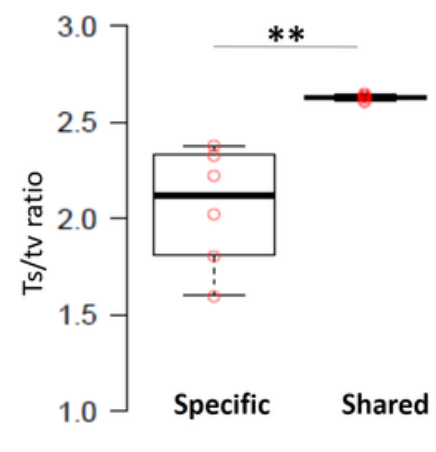




\section{2}

Figure 2

\section{Gene level comparison of SNPs specific in high and low milk-yield groups with QTL}

dataset: (A) Overlapping genes in milk-yield category. The first column represents high and low milk yield specific SNPs located in 26 common genes. The second and third column respectively indicates the 17 low-milk yield specific and 8 high-milk yield specific candidate genes (B) Overlapping genes in milk-fat category. The first column represents high and low milk yield specific SNPs located in 30 common candidate genes. The second and third column respectively indicates the 24 low-milk yield specific and 15 high-milk yield specific candidate genes.

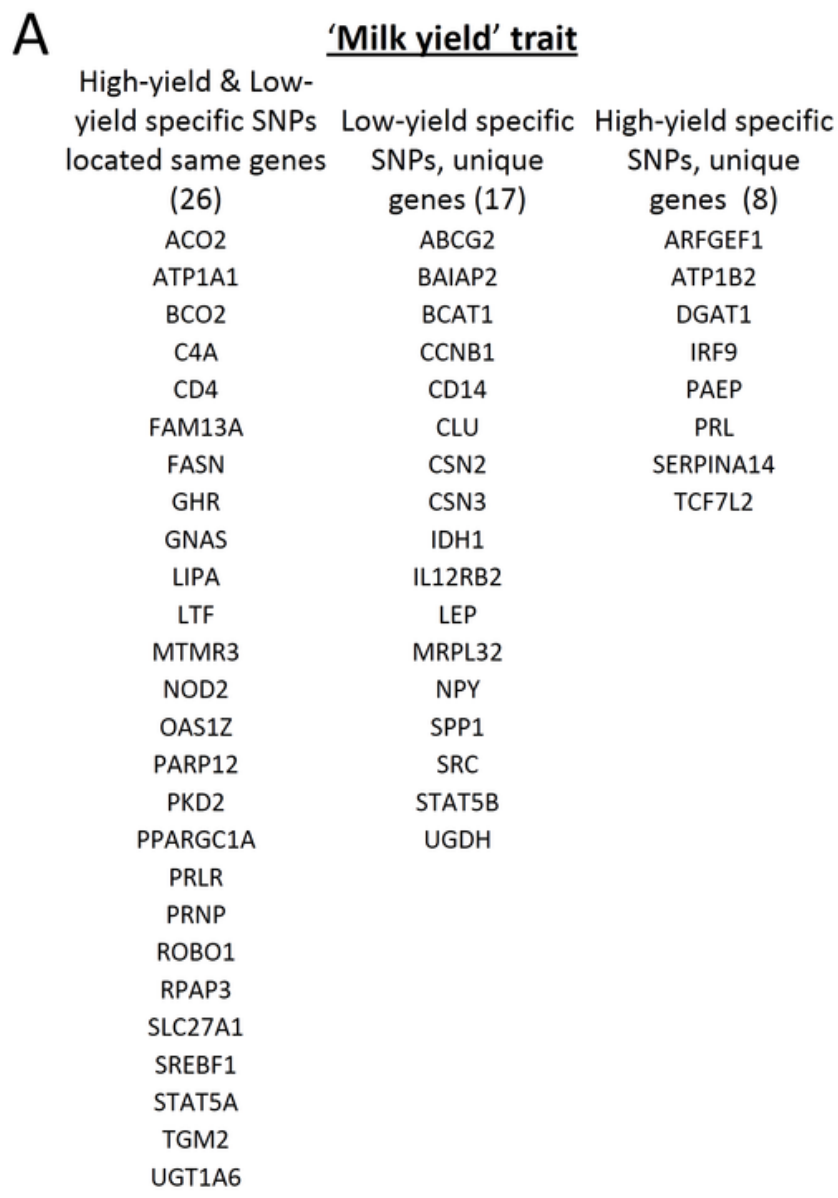

\begin{tabular}{|c|c|c|}
\hline \multicolumn{3}{|l|}{3} \\
\hline $\begin{array}{l}\text { High-yield \& Low- } \\
\text { yield specific SNPS }\end{array}$ & Low-yield specific & High-yield specific \\
\hline $\begin{array}{c}\text { located same genes } \\
\text { (30) }\end{array}$ & $\begin{array}{c}\text { SNPs, unique genes } \\
\text { (24) }\end{array}$ & $\begin{array}{c}\text { SNPs, unique genes } \\
\text { (15) }\end{array}$ \\
\hline $\mathrm{ACO} 2$ & $\mathrm{ABCG} 2$ & ARMC1 \\
\hline ANXA9 & APBA1 & ATP1B2 \\
\hline $\mathrm{BCO} 2$ & BAIAP2 & DGAT1 \\
\hline $\mathrm{C} 4 \mathrm{~A}$ & BCAT1 & $\mathrm{GH} 1$ \\
\hline CNOT1 & CCNB1 & IRF9 \\
\hline FAM13A & CD14 & MBTPS2 \\
\hline FASN & CLU & MSTN \\
\hline GHR & CSN2 & NDRG4 \\
\hline GINS4 & CSN3 & PAEP \\
\hline GNAS & FCGR2 & PRL \\
\hline INSIG1 & HERC6 & SERPINA14 \\
\hline KSR2 & $\mathrm{IDH} 1$ & STAT1 \\
\hline LIPA & LAP3 & TCF7L2 \\
\hline LTF & LEP & TF \\
\hline MTMR3 & MED28 & TSPAN32 \\
\hline NOD2 & MRPL32 & \\
\hline NPC1 & OLR1 & \\
\hline OAS1Z & SCD5 & \\
\hline PARP12 & SERPINA1 & \\
\hline PKD2 & SLC27A6 & \\
\hline PPARGC1A & SPP1 & \\
\hline PRLR & SRC & \\
\hline PRNP & TLR4 & \\
\hline ROBO1 & UGDH & \\
\hline SCAP & & \\
\hline SDR16C5 & & \\
\hline SREBF1 & & \\
\hline STAT5A & & \\
\hline TGM2 & & \\
\hline UGT1A6 & & \\
\hline
\end{tabular}


Figure 3

\section{Gene ontology enrichment analysis of high and low specific gene candidates: $(A)$}

Twenty one gene ontology (molecular functions) terms enriched in low-yield specific group.

The number of hits associated with each term along with the FDR corrected p-value is given.

(B) Eight gene ontology (molecular functions) terms enriched in high-yield specific group. (C)

The transcriptional regulators (140 genes) highlighted by the GO analysis in low-yield group.

A

Enriched GO molecular function terms in low-yield specific group
GO:0000166: nucleotide binding (MF)
GO:0046872: metal ion binding (MF)
GO:0003700: sequence-specific DNA binding transcription factor activity (MF)
GO:0043565: sequence-specific DNA binding (MF)
GO:0042803: protein homodimerization activity (MF)
GO:0005524: ATP binding (MF)
GO:0044212: transcription regulatory region DNA binding (MF)
GO:0008270: zinc ion binding (MF)
GO:0016740: transferase activity (MF)
GO:0003723: RNA binding (MF)
GO:0042802: identical protein binding (MF)
GO:0016787: hydrolase activity (MF)
GO:0008092: cytoskeletal protein binding (MF)
GO:0016491: oxidoreductase activity (MF)
GO:0046983: protein dimerization activity (MF)
GO:0005125: cytokine activity (MF)
GO:0044325: ion channel binding (MF)
GO:0004674: protein serine/threonine kinase activity (MF)
GO:0008553: hydrogen-exporting ATPase activity, phosphorylative mechanism (MF)
GO:0005525: GTP binding (MF)
GO:0003677: DNA binding (MF)

B GO:0046872: metal ion binding (MF) GO:0005126: cytokine receptor binding (MF) GO:0000166: nucleotide binding (MF) GO:0008270: zinc ion binding (MF) GO:0005125: cytokine activity (MF) GO:0016829: lyase activity (MF) GO:0005515: protein binding (MF) GO:0005215: transporter activity (MF)
Genes FDR p-val.

259 9.67E-11

$241 \quad 2.09 E-10$

112 2.46E-06

89 3.90E-06

$61 \quad 2.64 \mathrm{E}-05$

$190 \quad 6.94 \mathrm{E}-05$

$31 \quad 7.83 \mathrm{E}-05$

$227 \quad 1.51 \mathrm{E}-04$

$57 \quad 1.81 \mathrm{E}-04$

$60 \quad 1.91 \mathrm{E}-04$

$37 \quad 1.97 \mathrm{E}-04$

$95 \quad 2.30 \mathrm{E}-04$

9.04E-04

9.21E-04

2.49E-03

2.89E-03

5.62E-03

5.87E-03

$6.32 \mathrm{E}-03$

9.80E-03

$9.88 \mathrm{E}-03$

Genes FDR p-val.

$176 \quad 4.67 \mathrm{E}-10$

15 4.54E-06

$169 \quad 6.34 \mathrm{E}-06$

$162 \quad 2.01 \mathrm{E}-04$

$22 \quad 2.83 \mathrm{E}-04$

15 8.20E-04

$14 \quad 3.34 \mathrm{E}-03$

$29 \quad 3.85 \mathrm{E}-03$
C Transcriptional regulators in low yield specific group (140) $\begin{array}{lllll}\text { ADNP } & \text { ETS1 } & \text { IRF4 } & \text { NR1D1 } & \text { SMAD5 }\end{array}$ $\begin{array}{lllll}\text { ALX3 } & \text { FBXW7 } & \text { IRX3 } & \text { NR1H3 } & \text { SMAD6 }\end{array}$ $\begin{array}{lllll}\text { AR } & \text { FOSB JDP2 } & \text { NR113 SMARCA2 }\end{array}$ ARNTL FOSL1 JUN NR2E1 SNAI1 $\begin{array}{lllll}\text { ASH2L } & \text { FOSL2 } & \text { KLF1 } & \text { PBX4 } & \text { SPI1 }\end{array}$ $\begin{array}{lllll}\text { ATF2 } & \text { FOXI2 } & \text { KLF15 } & \text { PHB } & \text { STAT2 }\end{array}$ ATF3 FOXJ1 KLF4 PITX2 STAT6 $\begin{array}{lllll}\text { ATF4 } & \text { FOXJ2 } & \text { LHX3 } & \text { POLE4 } & \text { STRN3 }\end{array}$ BARHL2 FOXO4 LMX1A POU3F2 T BATF2 FOXP1 MEF2A PPARA TAF5 BSX GATA2 MEF2C PPARD TBX2 C1ORF85 GATA4 MEN1 PRDM1 TCF3 CALCOCO1 GBX2 MEOX1 PROX1 TFAP2A CDK9 GFI1 MGC140080 PURA TFE3 CEBPE GLI MITF RARA TLX1 $\begin{array}{llll}\text { CREB1 GLIS2 MNT RARG TSC22D4 } & \end{array}$ CREB3L3 GMEB1 MTERFD3 RAX2 USF1 CSRNP2 HESX1 MTF1 RBPJL VAX1 CSRNP3 HEY2 NANOG RCOR2 WNTSA CTNNB1 HIF1A NFATC1 RELA XRCC6 DDIT3 HOXA3 NFE2 RORB ZKSCAN1 DMRT2 HOXB1 NFE2L2 RUNX1T1 ZNF148 $\begin{array}{lllll}\text { E2F1 HOXB2 NFIB } & \text { RXRB } & \text { ZNF192 }\end{array}$ E2F2 HOXB9 NFIC $\quad$ RXRG ZNF263 E2F3 HOXC12 NFIL3 SCAND1 ZNF35 $\begin{array}{lllll}\text { ELF2 } & \text { HOXC13 NFX1 } & \text { SIX5 } & \text { ZNF367 }\end{array}$ ELF4 HOXD12 NFYB SMAD1 ZNF483 $\begin{array}{llll}\text { ELK3 } & \text { HSF1 NKX2-2 } & \text { SMAD4 } & \text { ZNF513 }\end{array}$ 


\section{4}

Figure 4

Comparison between DEG in high vs low-milk yield cattle and SNP candidate genes from buffalo breeds: The upper panel shows the workflow for the derivation of the DEG in high and low milk-yielding cattle using Agilent microarrays. The Venn diagram highlights the 582 DEG (55.1\% of total) which are discovered SNP candidate genes discovered by our multibreed study in buffaloes. The colored bar in indicates the group of SNP candidates in each category. Green color indicates 247 candidate genes common to high and low-yield specific groups, derived from specific SNPs in both the groups. Yellow and red color indicates the specific high-yield (139) and low-yield (196) candidate genes respectively. The overlap of the DEG and in each of these category were found to statistically significant in Hypergeometric test (p.val < 0.005). 
Agilent-023647 B. taurus v2 arrays (45220 probes)

High milk-yield (4 samples)
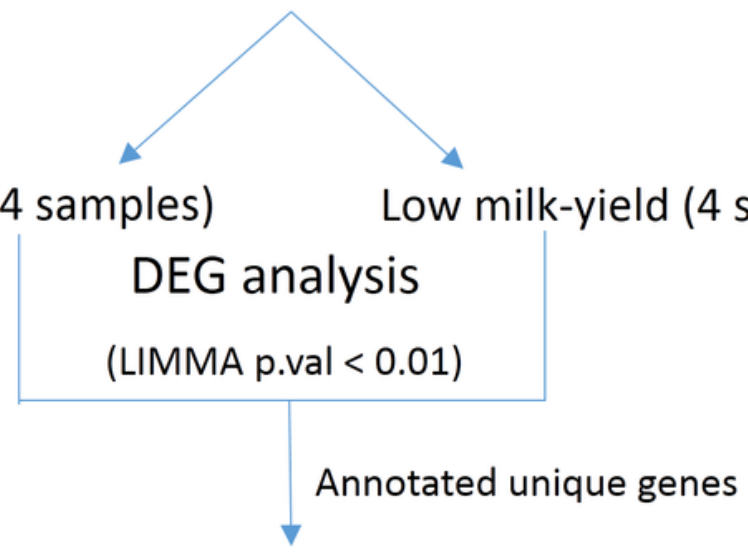

\section{DEG}

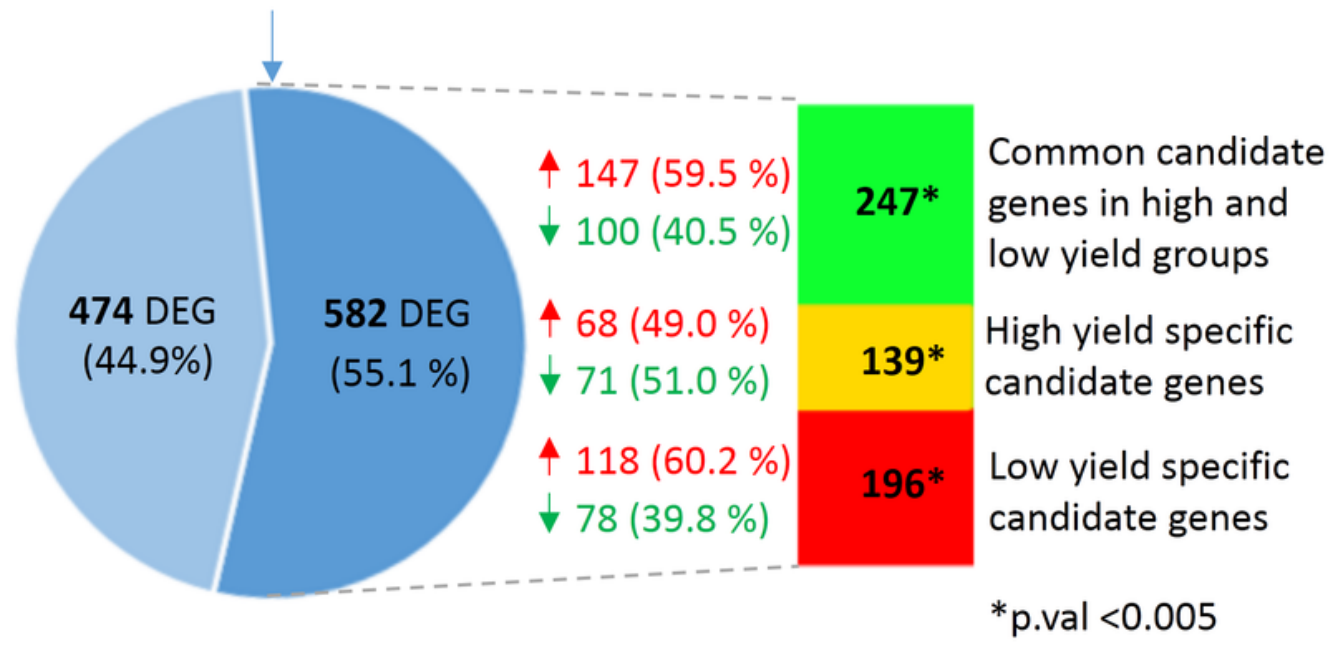

